\title{
Erratum
}

\section{Erratum: de la Vega et al., "Large-Scale Meta-Analysis of Human Medial Frontal Cortex Reveals Tripartite Functional Organization"}

In the article "Large-Scale Meta-Analysis of Human Medial Frontal Cortex Reveals Tripartite Functional Organization" by Alejandro de la Vega, Luke J. Chang, Marie T. Banich, Tor D. Wager, and Tal Yarkoni, which appeared on pages 6553- 6562 of the June 15, 2016 issue, three typographical errors were made in the typesetting stage. Any instances of "0-cluster" should read as "9-cluster." In particular, "... subregions in the 0 -cluster solution were given alphanumeric labels ..." should read as "... subregions in the 9-cluster solution were given alphanumeric labels."; "For the 0-cluster solution, we contrasted the coactivation of each cluster ..." should read as "For the 9-cluster solution, we contrasted the coactivation of each cluster..."; and "The 0-cluster solution revealed ..." should read as "The 9-cluster solution revealed ..." These corrections do not affect the conclusions of the paper.

DOI: 10.1523/JNEUROSCI.0471-17.2017 